\title{
TESTS OF THE PHOTOMETRIC ACCURACY OF IMAGE RESTORATION USING THE MAXIMUM ENTROPY ALGORITHM
}

\author{
JUDiTh G. COHEN \\ Palomar Observatory, California Institute of Technology, Mail Code 105-24, Robinson, Pasadena, California 91125 \\ Received 21 August 1990; revised 18 October 1990
}

\begin{abstract}
Simulations are described which test the maximum entropy image restoration algorithm as implemented in the MEMESYS 3 code (version 2) of S. F. Gull and J. Skilling [Quantified Maximum Entropy, MEMSYS3 Users' Manual, version 2.0 (1989) ]. It is found that at the faintest brightness levels, while the code can recover blurred point sources, they are recovered systematically too faint. The size of the photometric error depends on the brightness of the source (relative to the noise of the background) and the crowding of the field. The error increases substantially as the crowding increases. At present, the optimum technique to apply to blurred images of crowded fields where most of the sources are point sources seems to be to use a restored image to generate the list of objects, then feed this into a standard point-spread function-fitting code (such as DAOPHOT) and use this on the original blurred frame. In that manner, the most crowded fields can be analyzed without losing photometric accuracy. Additional simulations were carried out for images with the actual point spread function of the Wide Field Camera of the Hubble Space Telescope. For single isolated sources seen against a background characterized by Gaussian noise, the detection limit is degraded near its faint limit by a factor of about 4 compared to that expected. But reliable photometry cannot be obtained for sources at the detection limit in either the simulated frames, or those frames passed through the MEMSYS 3 image restoration code. If one requires photometry accurate to $10 \%$, the performance of the as-built $H S T$ plus Wide Field Camera is degraded near its faint limit by a factor of between 10 and 15 compared to that expected, even for isolated point sources.
\end{abstract}

\section{INTRODUCTION}

The image of a point source as seen in an astronomical telescope is blurred by several effects. [See Woolf (1982) for a useful review.] The first is diffraction, which gives an Airy pattern. The next limitation is that imposed by the Earth's atmosphere, which does not apply to spaceborne instruments such as the Hubble Space Telescope (HST). Then there are local effects such as thermal problems in the telescope enclosure, as well as optical aberrations resulting from imperfect fabrication, design, alignment, or focusing of the optical system. There are also guiding errors from imperfect drive systems or star trackers. In the worst case, the pointspread function may vary across the field of view of the detector, even when the field is quite small.

Minimizing the size of the point-spread function (psf) is extremely important. In the faint object case, where the dominant source of noise is the sky background, the signalto-noise ratio for direct imaging is proportional to $D / \theta$, where $D$ is the diameter of the aperture stop, so larger images are effectively reducing the telescope's collecting area, which is so expensive to build in the first place.

Many image restoration and image reconstruction algorithms have been developed over the past few decades. Bracewell (1979) and Pearson \& Readhead (1984) review their application to radio astronomy, while Narayan \& Nityananda (1986) discuss the maximum entropy method. The recent review issue of Optical Engineering with a preface by Sezan \& Tekalp (1990) focuses largely on medical applications, with emphasis on tomography. Although there have been a few advocates of such schemes (Arp \& Lorre 1976, Sharp 1989), they have not found widespread use in optical astronomy in part because of the severe computational requirements for restoring even a small $(128 \times 128$ pixel $)$ image. But with the increasing speed of computers, it is now feasible to begin serious exploration of such techniques.

The simulations described use the maximum entropy al- gorithm as implemented by the MEMSYS 3 code (version 2) of Gull \& Skilling (1989) (see also Gull 1989; Skilling 1989). Throughout we treat only point sources as seen against a uniform background subject to Gaussian noise. The principal concerns we address in our simulations are how faint an object can be detected and how reliable are the brightness of objects in a restored image. We are particularly interested in comparing the performance of an image restoration code with the use of a multiple psf fitting code such as DAOPHOT (Stetson 1987) on the original blurred frames, as this is much less computationally demanding and more conceptually straightforward.

After some preliminary simulations are discussed in Sec. 2, we examine the specific case of the Wide Field Camera of the HST in Sec. 3. A summary of our results is given in the last section.

\section{SIMULATIONS OF STARS IN A CROWDED FIELD}

To simulate stars in a crowded field, various patterns were created, and convolved with a Gaussian with a 3 pixel FWHM. We assume that the psf is known perfectly. In practice, the images of many objects can be used to determine the psf, so that is a reasonable assumption. After the convolution, noise is applied to the whole image assuming that the noise is a Gaussian distribution with the fluctuations determined by the value in each pixel. Thus we explicitly assume that the image is background limited, and that the noise fluctuations in the background are those of a Gaussian. The brightness of point sources is measured relative to the rms noise per pixel in the background. The integrated brightness of an object used as input into the simulation is denoted by $I_{\text {in }}$, which the recovered brightness is $I_{\text {out }} . I_{\text {in }}$ for this set of simulations corresponds to a signal-to-noise ratio as defined above of 1100. Of course, depending on the width of the psf, the signal-to-noise ratio for the brightest pixel in the extended image of a point source is much smaller. 
Point sources were laid out in a rectangular grid of $7 \times 7$ objects, alternating sources of brightness $I_{\text {in }}$ with either $I_{\text {in }} / 10, I_{\text {in }} / 30$, or $I_{\text {in }} / 100$. The grid was evenly spaced with 9.1 pixels between objects. In successive trials, the grid was gradually uniformly compressed from a separation of just over 3 FWHM between objects to less than 1 FWHM. At all times the spacing deliberately did not coincide with either an integer or a half integer number of pixels. Looking at the simulated images, everything essentially overlapped at the smallest separation used, 2.1 pixels $(0.7$ FWHM) between objects.

Figure 1 shows the ratio of the mean brightness of the recovered objects versus the input value for the stars with brightness $I_{\text {in }}, I_{\text {in }} / 10$, and $I_{\text {in }} / 30$. Two procedures were tried. First DAOPHOT was applied to the original blurred simulations. This gave good results with accurate brightness measurements until the finding algorithm used in DAOPHOT could no longer separate objects. Then, photometry was attempted on the frames restored with MEMESYS 3 . Since the images in the restored frames are too sharp for any pointspread function-fitting routine to work (essentially all the light ends up in a single pixel for a point source whose original blurred image is centered on a particular pixel), a $3 \times 3$ box sum centered on the maximum for each object was used. This will give a lower limit to the brightness of an object. In extremely crowded conditions, where the objects are closer than 2 pixels ( 0.7 FWHM) apart, this will be an underestimate of the object's brightness.

Both DAOPHOT and MEMSYS do well at recovering the bright objects at the correct value. But the maximum entropy method systematically fails by recovering the fainter objects as too faint, and the situation is clearly a function of the degree of contrast with nearby bright objects and the crowding.

One way of proceeding in very crowded fields where DAOPHOT cannot find the objects correctly because of image overlap is to use the maximum entropy restoration to gener- ate the list of object positions, and then to feed that list into DAOPHOT's multiple object-fitting routines. A test of that conceptual scheme for two stars of equal brightness $I$ (in), whose separation is gradually decreased in successive trials, is shown in Fig. 2. DAOPHOT itself was used for the well separated cases. Once DAOPHOT's object finding algorithm failed to indicate that there were two objects present (which occurred at a separation of about 1.5 FWHM), the remaining cases were treated by inputting the location of the two objects. Then the brightnesses were recovered by using DAOPHOT's psf fitting routines, and recovery was good even for objects separated by less than 1/3 the FWHM ( 1 pixel).

\section{SIMULATIONS FOR THE WIDE FIELD CAMERA ON THE HUBBLE SPACE TELESCOPE}

Given the optical problems of Space Telescope, a set of simulations were done to explore the potential for image restoration using the as-built point-spread function of the Wide Field Camera on HST. An actual WFC image illustrating the psf was obtained from J. Hester of the WF/PC team. Again we assumed that the field was background limited with Gaussian noise. This is a valid assumption for long exposures with broad bandpass filters at optical wavelengths because of the zodiacal light and can be verified using the information in the Wide Field and Planetary Camera Instrument Handbook (1985).

The simplest possible case was examined first, namely detection of an isolated point source against the background. A range of brightness of the point source was tried from $I$ (in) $=1100$ to the point where the source was no longer detectable in either the original blurred image or the restored image. The expected psf for the WFC on the HST was taken as a Gaussian with FWHM $=1.5$ pixels, which is slightly worse than the prelaunch predicted performance.

Figure 3 compares the actual WFC with the pre-launch expected performance. In both cases, image restoration via MEMSYS3 was performed on the blurred images. For the as-

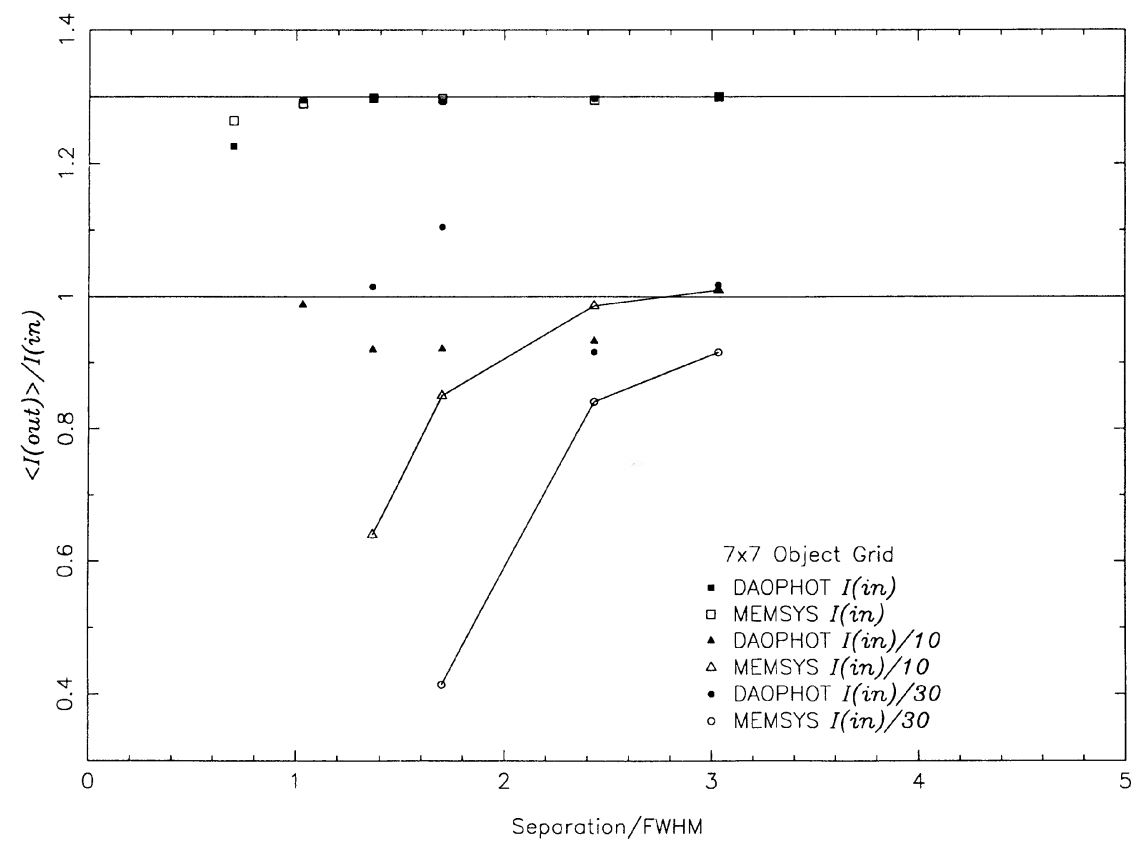

FIG. 1. In these simulations of the effect of crowding, the $x$ axis is the ratio of the separation of adjacent point sources in units of the FWHM of the Gaussian psf. The $y$ axis is the ratio of the brightness of the recovered image to that of the actual image. The results for stars of three different brightness levels (each adjacent to stars of the brightest level) are shown. The results for bright stars are offset by 0.3 along the $y$ axis for clarity. Open symbols denote simulations where the blurred image was first restored by the MEMSYS 3 code, then the brightness of the stellar objects was determined, while closed symbols denote simulations where the DAOPHOT code was used directly on the blurred image. 


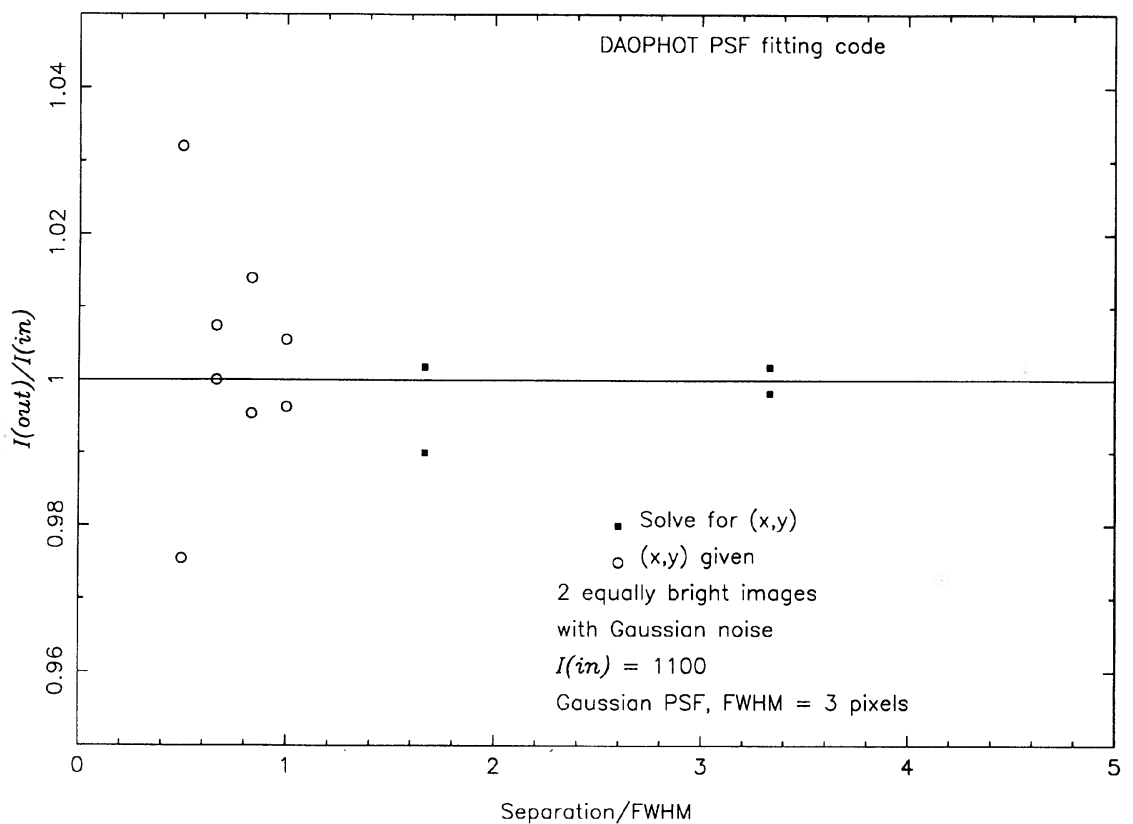

FIG. 2. The effect of crowding is illustrated by simulations of pairs of equally bright objects at various separations. DAOPHOT was used to analyze the original blurred frames. The axes are identical to those of Fig. 1. The closed symbols denote cases where the finding algorithm of DAOPHOT was used to locate each component of the pairs, while the open symbols denote cases where the location of the points was assumed given. built psf of the WFC, the light is so spread out that even with image restoration, reliable recovery does not occur once $I$ (in) $\leqslant 50$, and systematic photometric errors exceeding $10 \%$ occur at $I$ (in $) \leqslant 250$, always in the sense that the object is recovered too faint. In images with the expected psf, consistent recovery is achieved until $I$ (in $) \leqslant 12$, and photometric errors of under $10 \%$ are achieved for $I($ in $) \geqslant 25$. Here the photometric errors are due to the random fluctuations, rather than any systematic error due to use of the restoration procedure. The restoration procedure does not introduce systematic photometric errors at the $10 \%$ level until $I($ in $) \leqslant 15$. Furthermore, the rms fluctuation of the recov- ered sources at a given brightness level is about a factor of 34 smaller for images blurred with the sharp psf than for the actual as-built psf.

Note that the rms photometric errors of unrestored frames with the expected sharp psf using DAOPHOT are roughly comparable to those on the restored frames because the psf is so sharp.

Thus we find that in this highly idealized and simple case of an isolated point source if one merely asks for detection (i.e., is there a real point source at a particular location), the WFC on the actual as-built $H S T$ is degraded in performance by a factor of about 4 ( $1.5 \mathrm{mag}$ ). But if one requires photo-

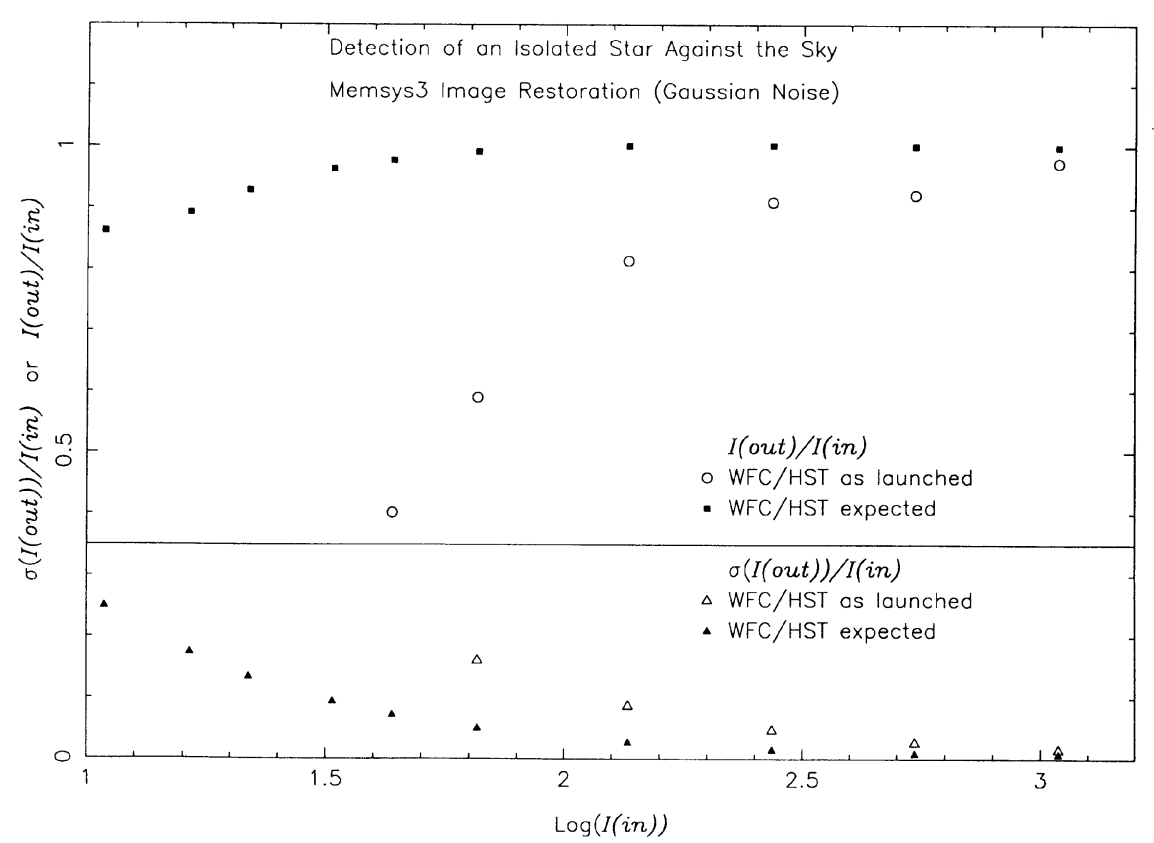

FIG. 3. Simulations of the detection of an isolated star against a background with Gaussian noise are shown for the WFC on the HST. The horizontal axis is the object brightness in units of the rms background noise pixel -1 , while the vertical axis is $I$ (out) $/ I$ (in) or $\sigma[I$ (out) $] / I$ (in). The open symbols are the instrument as launched, while the filled symbols denote the expected prelaunch performance. 
metric accuracy, the actual performance of the WFC on $H S T$ is degraded near the faint limit by a factor of between 10 and 15 ( 2.5 to $2.9 \mathrm{mag}$ ).

Simulations of closely spaced triplets of objects of varying brightnesses were also carried out. These show that because the actual WFC/HST image profile has a sharp core, image restoration can successfully separate sources which are 2 pixels apart. However a significant price is paid because of the degradation of the image profile over that expected. Faint sources [i.e., those with $I($ in $) \leqslant 50$ ] are not reliably recovered, and in addition to being recovered at systematically too faint magnitudes, the rms fluctuations in the brightness of those sources which are recovered is approximately 3 times that obtained for image blurred by the much sharper expected psf.

Sibisi (1989) investigated the validity of inversions of onedimensional emission line spectra using the maximum entropy algorithm as implemented by the MEMSYS 3 code. $\mathrm{He}$ found a similar problem, namely that the restored peaks of spectral lines are systematically low by about $2 / 3$ of the rms noise. This is probably related to the bias discussed by Narayan \& Nityananda (1986) and is just what we are finding. For a given psf used to blur the simulated images, the point sources in the images restored by the MEMSYS 3 code are systematically too faint by a particular value, and thus are proportionately more seriously affected as one looks at point sources closer to the detection limit. In the two-dimensional case, the value by which the restored point sources are too faint appears to be related to the rms noise of the background times the effective number of pixels in the psf and a factor which depends on the crowding.

\section{CONCLUSIONS}

We thus conclude that frames restored with a maximum entropy algorithm (at least that implemented the MEMSYS3 version 2.0 code) cannot be used where the photometric reliability of the result is critical. The best procedure where the photometric reliability is important is to use the restored frame to generate the object list, and then use a standard point-spread fitting routine like DAOPHOT to get the actual brightnesses. What to do in a situation where the image of interest is not that of a point source, yet the photometric accuracy of the result is important, and the dynamic range of the data is large, is not clear.

Compared to the prelaunch expected performance of the WFC on the HST, our simulations using the maximum entropy algorithm for image restoration show degradations of a factor of about 4 in the detection limit for point sources, but a degradation of a factor between 10 and 15 if one requires accurate $( \pm 10 \%)$ photometry.

I am grateful to Nick Weir and Steve Gull for implementing MEMSYS3 on our computers.

\section{REFERENCES}

Arp, H. C., and Lorre, J. 1976, ApJ, 210, 58

Bracewell, R. N. 1979, ARA\&A, 17, 113

Griffiths, R. 1985, Wide Field and Planetary Camera Instrument Handbook, NASA

Gull, S. F. 1989, in Developments in Maximum Entropy Data Analysis,

Maximum Entropy and Bayesian Methods, edited by J. Skilling,

(Kluwer, Dordrecht), pp. 53-71

Gull,. S. F. and Skilling, J. 1989, Quantified Maximum Entropy, MEMSYS3

Users' Manual, version 2.0

Narayan, R., and Nityananda, R. 1986, ARA\&A, 24, 127
Pearson, T. J., and Readhead, A. C. S. 1984, ARA\&A, 22, 97

Sezan, M. I., and Tekalp, A. M. 1990, Opt. Eng. 29, 391

Sharp, N. A. 1989, ApJ, 345, L37

Sibisi, S. 1990, in Quantified Maxent: An NMR Application, Maximum Entropy and Bayesian Methods, edited by J. Skilling (Kluwer, Dordrecht)

Skilling, J. 1989, in Classical Maximum Entropy, Maximum Entropy and Bayesian Methods, edited by J. Skilling (Kluwer, Dordrecht), pp. 45-52 Stetson, P. B. 1987, PASP, 99, 191

Woolf, N. J. 1982, ARA\&A, 20, 367 